\title{
RELACIÓN ENTRE ESTADO NUTRICIONAL Y SUEÑO EN ESCOLARES DE LA COMUNA DE SAN MIGUEL, SANTIAGO, CHILE
}

\author{
RELATIONSHIP BETWEEN NUTRITIONAL STATUS \\ AND SLEEP IN SCHOOL CHILDREN FROM THE SAN MIGUEL \\ COMMUNE, SANTIAGO, CHILE
}

\author{
Samuel Durán A. (1), Nineb Fuentes de la C. (1), Stephanie Vásquez Q. (1), \\ Gustavo Cediel G. (1), Víctor Díaz N. (2)
}

(1) Carrera de Nutrición y Dietética, Universidad Autónoma de Chile, Santiago, Chile.

(2) Facultad de Odontología. Universidad Finis Terrae. Santiago, Chile.

\begin{abstract}
Objective: To determine the association between sleep duration and obesity in school age children between 5 and 7 years old. Methods: One hundred fifty five children from schools in san Miguel town in Santiago of Chile participated in the study. Informed consent was obtained through their parents. Anthropometric variables were measured and a sleep questionnaire was applied. Results: Obesity was present in $19.3 \%$ of the subjects. $45.1 \%$ of the sample slept less than the accepted recommendation for their age (<10 hours). Sleeping more than 10 hours/ night, exercising, and not eating chocolate at night are associated factors for obesity $(\mathrm{OR}=0.7,0.1$ y 0.3 respectively), whereas sleeping less than 10 hours is an associated risk factor (OR=2.38). Conclusion: Sleeping less than the accepted recommended amount is an associated risk factor for obesity in this sample.
\end{abstract}

Keys words: Sleep, obesity, children.

Este trabajo fue recibido el 26 de Octubre de 2011 y aceptado para ser publicado el 15 de Enero de 2012.

\section{INTRODUCCIÓN}

Actualmente la obesidad es considerada como una epidemia de salud pública en todo el mundo (1). Según la Organización Mundial de la Salud (OMS), es una enfermedad crónica con alto riesgo de perpetuase cuando se adquiere desde la infancia y favorecer otras patologías como la diabetes tipo 2 y la cardiopatía isquémica arterioesclerótica $(2,3)$ primeras causas de muerte en la población chilena.

En Chile, la obesidad infantil se ha cuadruplicado en los últimos 20 años, constituyéndose en la enfermedad nutricional más importante en este grupo etareo. La alimentación saludable y la actividad física han sido el enfoque de investigación sobre prevención y tratamiento de la obesidad (4). Los resultados de las intervenciones han tenido poco éxito1, sólo algunos estudios han mostrado un impacto deseable sobre el IMC (5).

Dada la naturaleza multifactorial de la obesidad los expertos concuerdan en la importancia de reconocer y estudiar la implicancia de otros posibles determinantes ambientales; estudios previos han mostrado una asociación significativa entre la obesidad y el sueño $(6,7)$. Los mecanismos podrían estar relacionados con los cambios hormonales (leptina, grelina, insulina, cortisol y hormona del crecimiento) (8) durante la alteración del patrón del sueño normal, contribuyendo al desequilibrio energético, la sensibilidad a la insulina, el metabolismo de la glucosa y por consiguiente afectando la composición corporal $(9,10)$. Además, se ha descrito que las habilidades durante la vigilia del niño y el adulto están determinadas por la continuidad del proceso del sueño (8) dado su rol en procesos biológicos como la consolidación de la memoria, la respuesta inmunológica y la función neuroendocrina (9).

Dado los antecedentes resulta importante evaluar cual es la contribución del sueño a la elevada prevalencia de obesidad en los niños chilenos de 5 a 7 años de edad.

\section{SUJETOS Y MÉTODOS}

La muestra estuvo constituida por 155 alumnos de ambos sexos, que correspondían al 88\% de los alumnos 
entre 5 y 7 años de edad, de primer y segundo año básico de todas las escuelas municipales de la comuna de San Miguel, en Santiago de Chile. El 12\% restante de los alumnos no participó en el estudió porque no asistió a las evaluaciones o sus padres no aceptaron que su hijo(a) fuera evaluado. En sus respectivas escuelas, un grupo de estudiantes de tercer año de la carrera de Nutrición y Dietética realizó su evaluación antropométrica. A los padres de los escolares se les entrevistó y se les realizó un cuestionario sobre hábitos de sueño de su hijo(a), adaptado del cuestionario de sueño pediátrico validado en el Laboratorio de Sueño y Neurobiología Funcional del INTA, de la Universidad de Chile.

Las variables antropométricas estudiadas fueron peso, talla y pliegue cutáneo tricipital. La determinación del peso $(\mathrm{kg})$ se efectuó mediante una balanza de precisión marca SECA de 100 g mecánica, previamente calibrada con el mínimo de ropa posible (11). La estatura (cm) se obtuvo al medir al sujeto a través de un tallímetro marca SECA de precisión de $1 \mathrm{~mm}$ y portátil. El IMC se calculó dividiendo el peso por la talla al cuadrado $\left(\mathrm{IMC}=\right.$ peso $\mathrm{Kg} /$ talla $^{2}$ peso). El estado nutricional se clasificó según la norma técnica de evaluación del estado nutricional del niño de 6 a 18 años, considerando normal aquel escolar que se encontraba entre el p10 y $<$ p85, sobrepeso al escolar que estaba entre p85 y <p95 y con obesidad sobre el $\geq \mathrm{p} 95$. El pliegue tricipital se midió en el punto medio entre el acromion y olécranon del tríceps, utilizando un Cáliper marca Lange. Las medidas se realizaron por triplicado y se empleó la media de estos valores como el dato primario de las variables analizadas.

El cuestionario incluyó datos sobre comportamiento del sueño nocturno (presentaba o no ronquidos, bruxismo, sueño inquieto, movimientos corporales, entre otros), consumo de algunos alimentos, medicamentos para dormir y ejercicio físico que realizaban los escolares. Se consideró un escolar activo a aquel que realizar más de 30 minutos de ejercicio al menos por tres veces en la semana. El cuestionario fue aplicado a los padres. El consentimiento informado fue firmado por los padres previa explicación de los procedimientos. El estudio fue aprobado por el Comité de Ética de la Universidad Autónoma de Chile.

Entre los criterios de inclusión estaba ser escolares entre 5 y 7 años de edad de las escuelas municipales de la comuna de San Miguel y los criterios de exclusión correspondieron a aquellos escolares que no completaran la evaluación (antropométrica o la encuesta) y/o que presentarán trastornos de sueño en tratamiento con médico, y niños(as) que no vivían con sus padres.

Las variables independientes estudiadas en el presente trabajo fueron cantidad de sueño nocturno durante la semana, cantidad de sueño nocturno durante el fin de semana, presencia o ausencia durante el sueño de cefaleas, bruxismo, ronquido, sueño inquieto, sudoración, ir a orinar durante la noche, pesadillas frecuentes, dificultad para dormir y tipo de alimentos y medicamentos consumidos antes de dormir (después de las 20 horas). Las variables dependientes estudiadas en el presente trabajo fueron el estado nutricional clasificado según IMC/edad, y pliegue tricipital. El estado nutricional según IMC/edad se subdividió en 2 variables distintas: (a) Estado Nutricional 1, que incluyó en su clasificación 3 categorías de estado nutricional (normal, sobrepeso y obesidad) y (b) Estado Nutricional 2, que incluyó 2 categorías de estado nutricional (normal y sobrepeso/obesidad).

\section{ANÁLISIS DE DATOS}

Los datos fueron procesados en una planilla Excel. Para evaluar la normalidad de las variables continuas (edad, peso, entre otros) se utilizó la prueba de bondad de Shapiro-Wilk. Para variables que mostraron una distribución normal se utilizó la prueba estadística tStudent y para las variables no normales se utilizó la prueba U de Mann-Whitney. Se utilizaron distribuciones de frecuencia para las variables categóricas y prueba de $\chi^{2}$ para la comparación entre grupos.

Con el objeto de determinar la existencia de asociación entre las variables predictoras estudiadas en relación con las variables dependientes descritas anteriormente se empleó, en concordancia con las características de distribución de la variable dependiente (binomial o multinomial), una prueba de regresión logística de acuerdo con Visauta (12) y Hosmer y Lemeshow (13). También se estimó - 2 Log de verosimilitud (-2LL) con el objeto de comprobar la verosimilitud de los resultados obtenidos (14). Para conocer la magnitud de la influencia de la variable independiente sobre la variable dependiente (binomial), se estimaron el R2 de Cox y Snell y el R2 de Nagelkerke (12) y el pseudo R2 de Cox y Snell y Nagelkerke y McFadden (multinomial); en este último caso, la categoría de referencia fue la condición normal. Se estimó además el estadígrafo de Wald en todos los casos con el objeto de conocer la significación de los coeficientes de las variables en estudio (14). El nivel de significación utilizado fue de $\alpha=0,05$ en todos los casos. Los datos fueron procesados mediante el SPSS Package, versión 15.0; SPSS, Chicago, IL.

\section{RESULTADOS}

La muestra estudiada estuvo constituida por 155 escolares (56,7\% varones), la edad, peso y talla promedio fue de 6,6 $\pm 0,6$ años, 28,7 $\pm 6,7$ kilos, $1,23 \pm 0,06$ metros, respectivamente. El 50,3\% presentó un peso normal, $30,3 \%$ sobrepeso y el 19,3\% obesidad. Se observó que 
el $47,7 \%$ consumía bebidas cola en la noche, el $34.8 \%$ café o té y el $45.8 \%$ chocolates. El $45,1 \%$ de los escolares dormía menos de lo recomendado (10 horas), sin existir diferencias por sexo o por nivel educativo (primero a cuarto básico).

Al comparar los escolares con peso normal vs. sobrepeso/obesidad, estos últimos presentaron mayor talla, peso, IMC, pliegue tricipital y circunferencia de cintura $(\mathrm{p}<0,001)($ tabla 1$)$.

La cantidad de sueño los días de semana y de fin de semana no fue diferente entre los grupos, siendo 9,8 $\pm 1,1$ y $10,7 \pm 1,1$ en el grupo normopeso y $9,9 \pm 1,0$ y $10,2 \pm 1,2$ en el grupo sobrepeso/obesidad, sin embargo la diferencia entre la cantidad de horas de sueño durante la semana y la cantidad de horas de sueño del fin de semana, el grupo normopeso presenta una mayor diferencia que el grupo sobrepeso/obesidad $(\mathrm{p}<0,01)$.

Al comparar la prevalencia de alteraciones del sueño entre los grupos, la única diferencia fue mayor ronquido en el grupo de escolares obesos $66,1 \%$ vs $39,1 \%$ en los normopeso $(\mathrm{p}<0,01)($ tabla 2$)$.

La asociación entre las variables dependientes IMC y pliegue tricipital en relación con las variables independientes o predictoras examinadas no fue signi-

TABLA 1

Características generales de los estudiantes y cantidad de sueño durante la semana y fin de semana.

\begin{tabular}{|lccc|}
\hline Variables & Normales & SP/OB & valor p \\
\hline Edad (años)* $^{*}$ & $6,6 \pm 0,6$ & $6,6 \pm 0,7$ & 0,1 \\
Peso $(\mathrm{Kg})^{*}$ & $25,4 \pm 4,3$ & $32,0 \pm 6,5$ & 0,001 \\
Talla (mt) & $1,21 \pm 0,65$ & $1,25 \pm 0,63$ & 0,001 \\
IMC $\left(\mathrm{Kg} / \mathrm{mt}^{2}\right)^{*}$ & $17,0 \pm 2,0$ & $20,1 \pm 2,6$ & 0,001 \\
Pliegue tricipital (mm) & $10,0(7,2-12,0)$ & $14(11-19)$ & 0,001 \\
Perímetro cintura (cm) & $55,3(52,8-58,1)$ & $63(56,5-69,2)$ & 0,02 \\
Horas sueño semana (horas) ${ }^{\dagger}$ & $9,8(9,4-10,2)$ & $9,9(9,4-10,4)$ & 0,32 \\
Horas de sueño FDS (horas) & $10,9(9,9-11,7)$ & $10,2(9,4-11)$ & 0,06 \\
Diferencia hora sueño semana-hora sueño fin de semana* & $-0,94 \pm 1,3$ & $-0,40 \pm 1.2$ & 0,01 \\
\hline "Prueba T de Student (media \pm DE) & & & \\
${ }^{\dagger}$ Prueba Mann-Whitney U (mediana y rango intercuartil) & & & \\
\hline
\end{tabular}

TABLA 2

Comparación de factores que pueden afectar el sueño según estado nutricional

\begin{tabular}{|lccc|}
\hline Variables & Normal (77) & $\begin{array}{c}\text { Estado nutricional } \\
\text { Sobrepeso (47) }\end{array}$ & Obesidad (30) \\
\hline Roncar * & 39,1 & 33,3 & 61,1 \\
Movimientos de piernas & 34,7 & 56,6 & 50,0 \\
Bruxismo & 34,7 & 26,6 & 16,6 \\
Sueño inquieto & 35,1 & 36,2 & 60,0 \\
Transpirar al dormir & 42,9 & 51,1 & 53,3 \\
Pesadillas & 23,4 & 23,4 & 33,3 \\
Dificultad para dormir & 14,3 & 17,0 & 16,7 \\
Cefaleas al despertar & 5,2 & 8,5 & 6,7 \\
\hline$*$ Prueba $\mathrm{X}^{2} \mathrm{p}<0,01$ & & \\
\hline
\end{tabular}


ficativa $(\mathrm{p}>0,05)$.

En el tabla 3 se presentan los resultados de la prueba de regresión logística múltiple realizada para evaluar la variable dependiente estado nutricional 1. La categoría de referencia no mostró diferencias significativas en las variables predictivas evaluadas en los sujetos con sobrepeso, pero si se observaron diferencias significativas en las variables práctica de ejercicios y horas de sueño de fin de semana $(\mathrm{p}<0,05)$. Por lo tanto, la condición de obesidad está asociada con la ausencia de práctica de ejercicios y una cantidad de sueño reducida el fin de semana en relación con la variable dependiente estado nutricional 1. Los valores de ODDS RATIO fueron de 0,732 y 0,268 , todos con intervalos de confianza bajo el valor 1 .

En la tabla 4 se presentan los resultados de la

\begin{tabular}{|c|c|c|c|c|c|}
\hline \multicolumn{6}{|c|}{$\begin{array}{l}\text { Resultados de la asociación entre la variable dependiente Estado Nutricional } 1 \\
\text { y las variables predictoras estudiadas. }\end{array}$} \\
\hline \multicolumn{2}{|c|}{ Estado nutricional 1(a) } & \multirow[t]{2}{*}{ OR } & \multicolumn{2}{|c|}{ IC al $95 \%$} & \multirow{2}{*}{$\begin{array}{r}\text { Valor-p } \\
0,448\end{array}$} \\
\hline Sobrepeso & Intersección & & & & \\
\hline & Consumo de chocolate & 1,940 & 0,907 & 4,150 & 0,088 \\
\hline & Ejercicio & 1,426 & 0,602 & 3,376 & 0,420 \\
\hline & Ejercicios semana & 1,061 & 0,887 & 1,270 & 0,516 \\
\hline & Pesadillas & 1,021 & 0,438 & 2,378 & 0,962 \\
\hline & Pesadillas v/semana & 0,966 & 0,600 & 1,556 & 0,888 \\
\hline & DPD & 0,734 & 0,263 & 2,045 & 0,554 \\
\hline & Cefaleas & 2,262 & 0,553 & 9,253 & 0,256 \\
\hline & $\mathrm{N}^{\circ}$ cefaleas/sem & 1,117 & 0,332 & 3,757 & 0,858 \\
\hline & Bebidas cola & 0,936 & 0,417 & 2,098 & 0,872 \\
\hline & Café/té & 1,073 & 0,533 & 2,160 & 0,844 \\
\hline & Horas de sueño fin de semana $=1$ & 0,634 & 0,281 & 1,429 & 0,271 \\
\hline & Dormir $<10$ horas & 1,317 & 0,513 & 3,378 & 0,567 \\
\hline \multirow[t]{15}{*}{ Obesidad } & Intersección & & & & 0,714 \\
\hline & Consumo de chocolate & 2,637 & 0,902 & 7,708 & 0,076 \\
\hline & Ejercicio & 0,491 & 0,161 & 1,496 & 0,211 \\
\hline & Ejercicio semana & 0,732 & 0,560 & 0,955 & 0,022 \\
\hline & Pesadillas & 0,515 & 0,200 & 1,323 & 0,168 \\
\hline & Pesadillas v/semana & 0,794 & 0,442 & 1,429 & 0,442 \\
\hline & Dificultad para dormir & 0,936 & 0,261 & 3,357 & 0,920 \\
\hline & Cefaleas & 2,639 & 0,381 & 18,276 & 0,326 \\
\hline & $\mathrm{N}^{\circ}$ cefaleas/sem & 3,560 & 0,318 & 39,822 & 0,303 \\
\hline & Bebidas cola & 0,754 & 0,245 & 2,319 & 0,623 \\
\hline & Café/té & 0,747 & 0,303 & 1,838 & 0,525 \\
\hline & Hora sueño fin de semana $=1$ & 0,268 & 0,084 & 0,852 & 0,026 \\
\hline & Hora sueño fin de semana $=2$ & . & . & . & . \\
\hline & Dormir $<10$ horas & 3,162 & 0,912 & 10,964 & 0,070 \\
\hline & Dormir $>10$ horas & . & . & . & . \\
\hline
\end{tabular}


prueba de regresión logística binomial en la variable dependiente estado nutricional 2. Los resultados fueron significativos $(p<0,05)$ para las variables horas de sueño de fin de semana y consumo de chocolate. El primero se asocia positivamente al estado nutricional 2; es decir, existe un mejor estado nutricional si se duerme extendidamente el fin de semana y el consumo de chocolate es bajo. Los valores de "ODDS RATIO" fueron de 2,38 y 0,346 respectivamente. En el primer caso, el intervalo de confianza se sitúa por encima del valor de 1 , lo cual indica que los niños que duermen menos tienen 2,38 veces más posibilidades de tener un mal estado nutricional, mientras que no consumir chocolate en la noche se transforma en un factor protector de tener un inadecuado estado nutricional.

Por último, en la tabla 5 se muestra la capacidad de clasificar correctamente a los sujetos a partir de las variables estudiadas como predictoras de la condición de estado nutricional 2 . Se observa que en general este modelo logró clasificar correctamente al 71,1\% de los sujetos.

\section{DISCUSIÓN}

La restricción de sueño es cada vez más frecuente tanto en niños como en adultos (7). Estudios epidemiológicos en población pediátrica en diferentes países han encontrado evidencia de la existencia de relación entre cantidad de sueño y obesidad $(7,15)$.

Aunque en el presente estudio no hubo diferencias en la cantidad de sueño en la semana y el fin de semana, se observó una tendencia a que los estudiantes con estado nutricional normal duermen más extendidamente el fin de semana que los estudiantes con sobrepeso/obesidad $(\mathrm{p}<0,06)$, sugiriendo que los estudiantes eutróficos compensan más la restricción de sueño de los días de semana.

La cantidad de sueño inferior a 10 horas fue un factor de riesgo para obesidad. Chen (7) observó que los niños con menor duración de sueño ( $<10$ horas)

TABLA 4

\section{Resultados de la asociación entre la variable dependiente Estado Nutricional 2 y las variables predictoras estudiadas.}

\begin{tabular}{|lcccc|}
\hline & OR & \multicolumn{2}{c}{ I.C.95,0\% } & valor p \\
\hline Hora sueño fin de semana $<10 \mathrm{~h}$ & 2,384 & 1,081 & 5,255 & 0,031 \\
Hora sueño dicotomizado & 0,574 & 0,236 & 1,396 & 0,221 \\
Pesadillas & & & 0,392 \\
$\quad$ Sí & 0,321 & 0,015 & 6,812 & 0,466 \\
$\quad$ No & 0,604 & 0,040 & 9,213 & 0,717 \\
$\mathrm{~N}^{\circ}$ pesadillas/ semana & 0,721 & 0,411 & 1,266 & 0,255 \\
Cefaleas & & & & 0,517 \\
Sí & 0,035 & 0,000 & 26,233 & 0,321 \\
$\quad$ No & 0,600 & 0,110 & 3,270 & 0,555 \\
$\mathrm{~N}^{\circ}$ Cefaleas/semana & 1,816 & 0,764 & 4,319 & 0,177 \\
Bebidas cola & 0,785 & 0,362 & 1,701 & 0,539 \\
Consumo café/té & & & & 0,328 \\
Sí & 0,296 & 0,036 & 2,423 & 0,256 \\
$\quad$ No & 1,299 & 0,582 & 2,897 & 0,523 \\
Consumo chocolate & & & 0,041 \\
$\quad$ Sí & 0,712 & 0,085 & 5,971 & 0,754 \\
No & 0,346 & 0,149 & 0,803 & 0,014 \\
Ejercicio & & & 0,831 \\
Sí & 0,444 & 0,007 & 28,677 & 0,702 \\
No & 0,316 & 0,025 & 4,028 & 0,375 \\
\hline
\end{tabular}


tenían un 58\% de mayor riesgo de sobrepeso y obesidad (OR combinado $=1,58 ; 95 \%$ IC: 1,26 a 1,98$) 7$. Por cada hora más de sueño, el riesgo de sobrepeso/obesidad se reducía $9 \%(\mathrm{OR}=0,91 ; 95 \%$ IC 0,84 a 1). Una revisión reciente resume trece estudios que examinaron la asociación entre cantidad del sueño y obesidad en niños y adolescentes, y recomienda dormir lo adecuado para evitar la obesidad (7).

Por otra parte, nuestro estudio no mostró diferencias por sexo como si lo han evidenciado Chen y col. (7) y Sekine y col. (8), donde la asociación entre cantidad del sueño y sobrepeso/obesidad fue mayor en niños que en niñas (la OR ajustada con 9-10 horas fue de 2,28, mientras que sólo fue de 1,49 con 10 o más horas de sueño). Un estudio en niños australianos encontró una relación inversa entre cantidad de sueño y sobrepeso/obesidad en niños pero no en niñas (16). Algunos investigadores desde una perspectiva evolutiva sostienen que las niñas podrían ser más resistentes a los factores ambientales generadores de estrés (17.) Más estudios son necesarios para aclarar la diferencia por sexo.

Además de la restricción de sueño, la actividad física reducida y el consumo de chocolates en la noche se asociaron con mayor riesgo de obesidad. En relación al chocolate, tal asociación podría explicarse por el hecho que éste contiene grasa y cafeína (cacao), este último es un alcaloide estimulante del sistema nervioso central (18). Otros alimentos que contienen cafeína (té, café y bebidas cola), cuyo consumo era frecuente en los escolares evaluados, no mostró ser un factor de riesgo en la reducción del sueño.

La restricción de sueño puede influir en el desarrollo de la obesidad a través de diversas vías biológicas, incluyendo un mayor tono simpático, aumento de la concentración plasmática de cortisol y de grelina y dis- minución de la de leptina y hormona de crecimiento y, por último, alteración de la tolerancia a la glucosa (19).

Spigel y cols. mostraron que una restricción aguda de la cantidad de sueño nocturno (4 horas por 6 noches), disminuye la concentración plasmática de leptina en adultos jóvenes y sanos. Un estudio posterior confirmó dichos hallazgos además de mostrar un incremento de la concentración plasmática de grelina en un $18 \%$, que es una hormona orexigénica y antagonista de leptina, que es anorexigénica (20). Estos cambios neuroendocrinos se han asociado con mayor sensación de hambre y apetito diurnos (20). Incluso, otros autores han mostrado que bastaría solo una noche de privación de sueño para que la concentración plasmática de grelina y la sensación de hambre diurna incrementen significativamente (21).

Estos cambios hormonales podrían contribuir a la selección y apetencia por alimentos densos en calorías (22) y, puesto que la restricción de sueño afecta la tasa metabólica basal, induce un aumento de citoquinas que desencadenan respuestas inflamatorias conducentes a enfermedades cardiovasculares (23).

En conclusión, los resultados obtenidos en el presente estudio muestran una asociación entre menor cantidad de sueño durante el fin de semana y aumento del riesgo de obesidad en la edad escolar. Estudios longitudinales son imprescindibles para abordar la dimensión de causalidad y, concurrentemente, para identificar los mecanismos biológicos involucrados en el desarrollo de la obesidad.

Los patrones de sueño serían un factor importante a considerar en la prevención de la obesidad infantil. Una combinación de estrategias dirigida a escolares para que se acuesten más temprano y duerman lo que corresponde, debiera ayudar a prevenir la obesidad infantil. En consecuencia, postulamos que, sumado a la alimentación

\section{TABLA 5}

Resultados de la clasificación a posteriori de los sujetos estudiados a partir de las variables predictoras estudiadas en la variable dependiente estado nutricional 2.

\begin{tabular}{|c|c|c|c|c|c|}
\hline & \multirow{2}{*}{\multicolumn{2}{|c|}{ Observado }} & \multicolumn{3}{|c|}{$\begin{array}{l}\text { Pronosticado } \\
\text { VD4 estado nutricional } 2\end{array}$} \\
\hline & & & Normal & Sobrepeso + Obeso & Porcentaje correcto \\
\hline \multirow[t]{2}{*}{ Paso 1} & $\begin{array}{l}\text { VD4 } \\
\text { Nutricional } 2\end{array}$ & Estado Normal & 54 & 22 & 71,1 \\
\hline & Porcentaje glo & $\begin{array}{l}\text { Sobrepeso + Obeso } \\
\text { al }\end{array}$ & 22 & 54 & $\begin{array}{l}71,1 \\
71,1\end{array}$ \\
\hline
\end{tabular}


sana y saludable y a la actividad física adecuada, tanto la cantidad (idealmente también la calidad) como los horarios de sueño debieran de ser considerados al diseñar intervenciones para combatir la obesidad.

\section{RESUMEN}

Objetivo: Determinar si existe asociación entre una menor cantidad de horas de sueño y la obesidad en escolares entre 5 y 7 años. Métodos: Se estudiaron 155 escolares de primer y segundo año básico de las escuelas municipales de la comuna de San Miguel, Santiago de Chile, a los cuales se les realizó una evaluación antropométrica y a sus padres una encuesta sobre hábitos de sueño de su hijo(a). Resultados: El 19,3\% de los escolares presentó obesidad. El 45,1\% de los escolares duerme menos de lo recomendado ( $<10$ horas). Dormir más, hacer ejercicios ( $\mathrm{OR}=0,7$ y 0,1 , respectivamente) y evitar el consumo de chocolate en la noche $(\mathrm{OR}=0,346)$, fueron factores protectores asociado a la presencia de obesidad en la muestra estudiada. En cambio, dormir menos de 10 horas $(\mathrm{OR}=2,38)$ es un factor de riesgo para obesidad en la muestra estudiada. Conclusión: La restricción de sueño es un factor de riesgo asociado con la presencia de obesidad en la muestra estudiada.

Palabras clave: sueño, obesidad, niños.

Conflicto de intereses: Los autores declaran que no hay conflicto de intereses de ningún tipo en el estudio.

Agradecimientos: Agradecemos a todos los docentes que colaboraron con su valioso tiempo en revisar este trabajo y entregar sus valiosas sugerencias.

Dirigir la correspondencia a:

Profesor

Samuel Durán A.

Carrera de Nutrición y Dietética

Facultad de Salud

Universidad Autónoma de Chile

Santiago, Chile

Email:sduran74@gmail.com

\section{BIBLIOGRAFÍA}

1. Sharma M. International school-based interventions for preventing obesity in children. Obes Rev 2007; 8:155-67.

2. Seng Y. Consequences of childhood obesity. Ann Acad Med Singapore 2009; 38:75-81.

3. Olivares S, Zacarías I, Lera L, Leyton B, Durán R, Vio F. Estado nutricional y consumo de alimentos seleccionados en escolares de la Región Metropolitana: Línea base para un proyecto de promoción del consumo de pescado. Rev Chil Nutr 2005; 32:102-8.

4. Mendelson R. Think tank on school-aged children: nutrition and physical activity to prevente the rise in obesity. Appl Physiol Nutr Metab 2007; 32:495-9.

5. Summerbell CD, Waters E, Edmunds LD et al. Interventions for preventing obesity in children. Cochrane Database Syst Rev 2005; CD001871.

6. Eisenmann JC. Insight into the causes of the recent secular trend in pediatric obesity: common sense does not always prevail for complex, multi-factorial phenotypes. Prev Med 2006; 42:329-35.

7. Chen X, Beydoun MA, Wang Y. Is sleep duration associated with childhood obesity? A systematic review and meta-analysis. Obesity (Silver Spring) 2008; 16:265-74.

8. Sekine M, Yamagami T, Handa $\mathrm{K}$ et al. A doseresponse relationship between short sleeping hours and childhood obesity: results of the Toyama Birth Cohort Study. Child Care Health Dev 2002; 28:163-70.

9. Spiegel K, Leproult R, L'Hermite-Baleriaux M et al. Leptin levels are dependent on sleep duration: relationships with sympathovagal balance, carbohydrate regulation, cortisol, and thyrotropin. J Clin Endocrinol Metab 2004; 89:5762-71.

10. Gibson S, Lambert J, Neate D. Associations between weight status, physical activity, and consumption of biscuits, cakes and confectionery among young people in Britain. Nutr Bulletin 2004; 29:301-9.

11. Muzzo S, Burrows R, Cordero J, Ramírez I. Trends in nutritional status and stature among school- age children in Chile. Nutrition 2004; 20: 867-72.

12. Visauta, B. Análisis estadístico con SPSS. McGraw-Hill. Madrid. 1998.

13. Hosmer, D. W., and S. Lemeshow. Applied Logistic Regression, 2nd ed. New York: John Wiley and Sons. 2000.

14. Lëvy, JP; Varela, J. Análisis Multivariable para las Ciencias Sociales. Prentice Hall. Madrid. 2003.

15. von Kries R, Toschke AM, Wurmser h, Sauerwald T, Koletzko B. Reduced risk for overweight and obesity in 5- and 6-y-old children by duration of sleep - a cross-sectional study. Int J Obes Relat Metab Disord 2002; 26:710-6.

16. Eisenmann JC, Ekkekakis P, Holmes M. Sleep duration and overweigth among Australian children and adolescents. Acta Paediatr 2006; 95:956-63.

17. Wells JC. Natural selection and sex differences in morbidity and mortality in early life. J Theor Biol 2000; 202:65-76.

18. Gotteland M, De Pablo S, Algunas verdades sobre el café. Rev Chil Nutr 2007; 34:105-15. 
19. Knutson KL, Spiegel K, Penev P et al. The metabolic consequences of sleep deprivation. Sleep Med Rev 2007; 11:163-78.

20. Spiegel K, Tasali E, Penev P, Van Cauter E. Brief communication: Sleep curtailment in healthy young men is associated with decreased leptin levels, elevated ghrelin levels, and increased hunger and appetite. Ann Intern Med 2004 Dec 7;141(11):846-50.

21. Schmid SM, Hallschmid M, Jauch-Chara K, Born J, Schultes B. A single night of sleep deprivation increases ghrelin levels and feelings of hunger in normal-weight healthy men. J Sleep Res 2008 Sep;17(3):331-4.

22. Van Cauter E, Knutson K. Sleep and the epidemic of obesity in children and adults. European J Endocrinol 2008;159:59-66.

23. Tauman R, Ivanenko A, O'Brien LM, Gozal D. Plasma C-reactive protein levels among children with sleep-disordered breathing. Pediatrics 2004; 113:564-9. 